\title{
Application of an improved targeted next generation sequencing method to diagnose non-syndromic mental retardation in one step: A case report
}

\author{
WEIPENG WANG $^{1 *}$, BING MAO $^{2 *}$, XIAOMING WEI $^{3}$, DAN YIN $^{3}, \mathrm{HUI} \mathrm{LI}^{1}$, \\ LIANGWEI MAO ${ }^{3,4}$, XUEQIN GUO ${ }^{3}$, YAN SUN ${ }^{3,5}$ and YUN YANG ${ }^{3,6}$ \\ ${ }^{1}$ Prenatal Diagnosis Center, Hubei Maternal and Child Health Hospital, Wuhan, Hubei 430070; ${ }^{2}$ Department of Neurology, \\ Wuhan Medical and Health Center for Women and Children, Wuhan, Hubei 430016; ${ }^{3}$ BGI-Wuhan, BGI-Shenzhen, \\ Wuhan, Hubei 430074; ${ }^{4}$ College of Life Sciences, Hubei University, Wuhan, Hubei 430062; ${ }^{5}$ Department of Biology, \\ University of Copenhagen, DK-1165 Copenhagen, Denmark; ${ }^{6}$ Department of Obstetrics and Gynecology,
}

The Second Affiliated Hospital of Zhengzhou University, Zhengzhou, Henan 450052, P.R. China

Received October 18, 2017; Accepted February 1, 2018

DOI: $10.3892 / \mathrm{mmr} .2018 .9031$

\begin{abstract}
The genetic basis of congenital mental retardation includes chromosomal anomalies and single gene mutations. In addition to chromosome microarray analysis, next-generation sequencing (NGS) and Sanger sequencing have additionally been applied to identify single gene mutations. However, no methods exist to identify the cause of an anomaly in one step. The present study applied an improved targeted NGS method to diagnose an 8 -year-old Chinese Han female with mental retardation in one step. The microdeletion $17 \mathrm{p} 11.2$ was successfully detected by the improved targeted NGS and no single gene mutations were identified. The same microdeletion was verified using low coverage whole-genome sequencing. Fertility guidance was also given to the patient's parents. In the present study, an improved targeted NGS method was applied to diagnose non-syndromic mental retardation of unknown cause in one step. This improved method has the potential to be developed into a screening panel for the effective diagnosis of genetic abnormalities in non-syndromic mental retardation and other congenital anomalies.
\end{abstract}

\section{Introduction}

Congenital mental retardation is a neurodevelopmental disease that affects $2-3 \%$ of the population worldwide $(1,2)$. Genetic

Correspondence to: Dr Yun Yang, BGI-Wuhan, BGI-Shenzhen, 666 Gaoxin Road, Wuhan East Lake High-Tech Development Zone, Wuhan, Hubei 430074, P.R. China

E-mail: yangyun@genomics.cn

*Contributed equally

Key words: non-syndromic mental retardation, Smith-Magenis syndrome, molecular diagnosis, improved targeted next generation sequencing, low coverage whole genome sequencing factors may explain $>50 \%$ of congenital mental retardation cases (3). Chromosomal anomalies, including chromosomal deletion, duplication and trisomy contribute to $4-28 \%$ of cases and known monogenetic diseases explain 3-9\% of cases (4). Various methods may be used to detect genetic abnormalities, including chromosomal microarray (CMA) and next generation sequencing (NGS) (5-10). However, the absence of any accompanying deformities in non-syndromic mental retardation makes it difficult to select the detection method. Using numerous methods increases cost and reduces efficiency. To the best of our knowledge, no method to detect genetic abnormalities in one step exists at present.

Typically, custom-made capture array-based targeted NGS is used to detect disease-causing mutations in monogenic disease (8). A custom-made capture array that is able to detect various types of mutations, including microdeletion, was previously designed (11). In the present study, this improved targeted NGS method was applied to diagnose a case of non-syndromic mental retardation of unknown cause. This may aid in the detection of genetic abnormalities in one step.

The microdeletion $17 \mathrm{p} 11.2$ was successfully detected by improved targeted NGS and no single gene mutations were identified. Subsequently, the same microdeletion was validated using low coverage whole-genome sequencing (LCS). Fertility guidance was additionally given to the parents of the patient.

In the present study, the patient was diagnosed with Smith-Magenis syndrome (SMS). SMS is a rare, congenital syndrome that affects $\sim 1$ in every 25,000 individuals. Features include mental retardation, behavioral abnormalities and distinctive facial features, difficulty sleeping, delayed speech and development, resulting from a 17p11.2 deletion encompassing the retinoic acid-induced protein 1 (RAI1) (12-14). It is estimated that $70 \%$ of patients with SMS have a common $3.7 \mathrm{Mb}$ deletion; the remaining $30 \%$ have larger or smaller deletions. Approximately $90 \%$ of SMS patients have a 17p11.2 deletion; the remaining $30 \%$ have mutations in the RAI1 gene (15). 

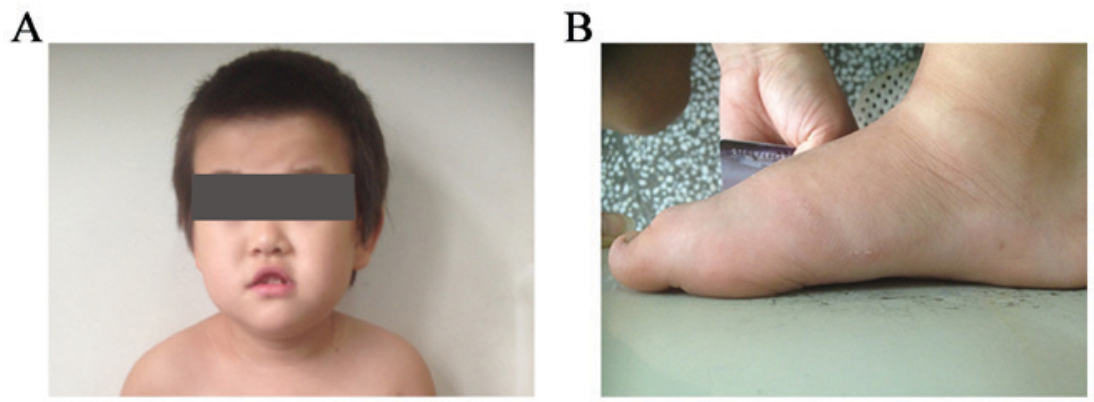

Figure 1. Distinct facial features of the patient. A typical Smith-Magenis syndrome phenotype was observed, with a (A) tented upper lip, depressed nasal bridge and (B) characteristic flat feet.

The present study applied an improved targeted NGS method to diagnose a patient with non-syndromic mental retardation of an unknown cause in an effective and a fast way, without using other methods. The result was validated by LCS. This improved method has the potential to be developed into a screening panel to effectively identify genetic abnormalities in non-syndromic mental retardation and other congenital anomalies.

\section{Case report}

Patient information. An 8-year-old Chinese Han female and her parents attended the Wuhan Medical \& Health Center for Women and Children hospital due to developmental delay and signs of mental retardation that were present from infancy. The research was prospectively reviewed and approved by the ethics committee of BGI-Shenzhen (approval no. BGI-IRB 15083; Shenzen, China). Written informed consent for participation in the present study was obtained from the patient's parents.

The patient was the first child of unrelated parents who had no family history of inherited diseases. The patient was born by cesarean section with a birth weight of $3.35 \mathrm{~kg}$ and a body length of $50 \mathrm{~cm}$. A distinct facial appearance was observed, with short palpebral fissures, a depressed nasal bridge, hypertelorism, and an upper lip with tented morphology and a V-like shape (Fig. 1A and B).

By age 2 years, the patient had sleep difficulties with nocturnal awakenings which gradually increased in frequency. The patient exhibited signs of mental retardation and decreased motor development compared with other children of the same age. The patient was talkative, although failed to convey information effectively.

Giemsa (G)-banded cytogenetic analysis. Routine G-banding chromosome analysis was performed as described (16); a $5 \mathrm{ml}$ peripheral blood sample was collected from the patient and her parents. Chromosomes from cultured lymphocytic cells were treated with $0.25 \%$ trypsin (Sigma-Aldrich; Merck KGaA, Darmstadt, Germany) for 2 min at $37^{\circ} \mathrm{C}$ and stained with $6 \%$ Giemsa (Sigma-Aldrich; Merck KGaA) for $10 \mathrm{~min}$ at $37^{\circ} \mathrm{C}$. The results revealed that the patient carried the normal karyotype (46 chromosomes; XX; Fig. 3). In addition, the parents of the patient carried the normal karyotype.

Improved targeted NGS. Improved targeted NGS was performed on peripheral blood samples of the patient. The

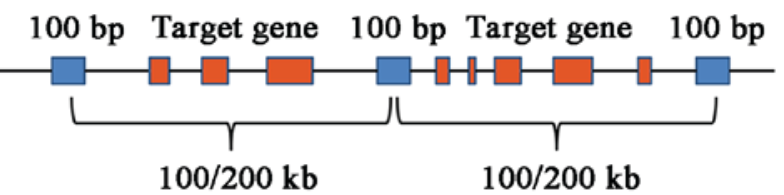

Figure 2. Target spot in the designed capture array for the detection of microdeletions and microduplications. The region that contained known microdeletions and microduplications was covered and a target spot at an interval of $0.1 \mathrm{Mb}$ (length, $100 \mathrm{bp}$ ) was selected; for the remaining genome region, a target spot at an interval of $0.2 \mathrm{Mb}$ (length, $100 \mathrm{bp}$ ) was selected.

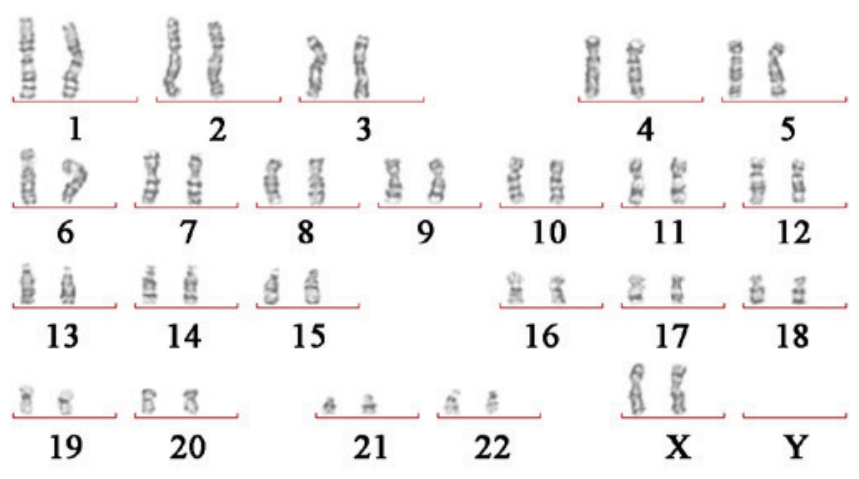

Figure 3. Patient karyotype. G-banding analysis revealed a normal chromosomal karyotype.

designed capture array (NimbleGen; Roche Molecular Diagnostics, Pleasanton, CA, USA) focuses on known associated single gene-coding regions and flanking intronic boundaries $10 \mathrm{bp}$. The region that contained known microdeletions and microduplications was covered and a target spot at an interval of $0.1 \mathrm{Mb}$ (length, $100 \mathrm{bp}$ ) was selected; for the remaining genome region, a target spot at an interval of $0.2 \mathrm{Mb}$ (length, 100 bp) was selected (Fig. 2). Targeted NGS analysis with normalization was performed for the patient (17). The microdeletion and microduplication regions designed on this array were selected from the database of DECIPHER (18). The 45 known microdeletion and microduplication diseases can be identified at present listed in Table I.

Sequence capture, enrichment and elution was performed according to the standard protocol, as previously mentioned (11). Following enrichment, high-throughput sequencing was performed using the Illumina Hiseq2000 analyzer (Illumina, 
Table I. Known chromosomal microdeletion or microduplication diseases.

Syndrome

Chromosomal region

$12 \mathrm{q} 14$ microdeletion syndrome

$15 q 13.3$ microdeletion syndrome

chr12: 65071919-68645525

$15 \mathrm{q} 24$ recurrent microdeletion syndrome

chr15: 30910306-32445407

15 q26 overgrowth syndrome

chr15: 74412643-75972911

16p11.2 microduplication syndrome

chr15: $99357970-102521392$

16p11.2-p12.2 microdeletion syndrome

chr16: $29606852-30199855$

16p11.2-p12.2 microduplication syndrome

chr16: $21512062-30199854$

$16 \mathrm{p} 13.11$ recurrent microdeletion or microduplication

chr16: $21475060-29284077$

$17 \mathrm{q} 21.31$ recurrent microdeletion syndrome

$1 \mathrm{p} 36$ microdeletion syndrome

chr16: $14986684-16486684$

chr17: 43705166-44294406

1q21.1 recurrent microdeletion or microduplication

1q21.1 susceptibility locus for Thrombocytopenia Absent Radius syndrome

chr1: 10001-12840259

22 q11 deletion or duplication syndrome

$22 q 13$ deletion syndrome

2p15-16.1 microdeletion syndrome

2p21 microdeletion syndrome

2q33.1 deletion syndrome

2q37 monosomy

$3 q 29$ microdeletion or microduplication syndrome

7q11.23 duplication syndrome

8p23.1 deletion or duplication syndrome

9q subtelomeric deletion syndrome

Angelman syndrome type 1

Angelman syndrome type 2

$\alpha$-thalassemia-intellectual deficit syndrome

Azoospermia factor microdeletion

Charcot-Marie-Tooth syndrome type 1A

Cri du Chat syndrome

Early-onset Alzheimer disease with cerebral amyloid angiopathy

Familial Adenomatous Polyposis

Miller-Dieker syndrome

NF1-microdeletion syndrome

Pelizaeus-Merzbacher disease

Smith-Magenis syndrome

Potocki-Shaffer syndrome

Renal cysts and diabetes

Rubinstein-Taybi syndrome

Sotos syndrome

Steroid sulphatase deficiency

11 p13 deletion syndrome

Wolf-Hirschhorn syndrome

chr1: $146533376-147883376$

chr1: 145386506-145748067

chr22: $19009792-21452445$

chr22: $51045516-51187844$

chr2: 59285696-61819815

chr2: 44410451-44589584

chr2: 196925121-205206939

chr2: 239969863-240322643

chr3: $195726835-197344663$

chr7: 72744455-74142672

chr8: 8100055-11764629

chr9: 140513443-140730578

chr15: 22749354-28438266

chr15: $23619912-28438266$

chr16: 60001-834372

Chr Y: 14352761-15154862

chr17: $14097915-15470903$

chr5: 10001-12533304

chr21: 27252860-27543446

chr5: 112043201-112181936

chr17: 1-2588909

chr17: 29107097-30263321

chrX: 103031438-103047547

chr17: $16773072-20222149$

chr11: 43994800-46052450

chr17: $34815072-36215917$

chr16: 3775055-3930121

chr5: 175724636-177052116

chrX: 6455812-8133195

chr11: 31806339-32457087

chr4: 1569197-2110236

Xp11.22-linked intellectual disability

Xp11.22-p11.23 microduplication

chrX: 53401070-53683275

chrX: 48334549-52117661

Xq28 duplication

chrX: 153287263-153363188

Xq28 microduplication

chrX: 153624563-153881853

Chr, chromosome.

Inc., San Diego, CA, USA). Image analysis and base-calling were performed using the Illumina Pipeline version 1.3.4 (Illumina, Inc.).
The depth ratio (patient:control) was used to evaluate the microdeletions and microduplications; the control referred to healthy subjects unrelated to the patient (samples obtained 


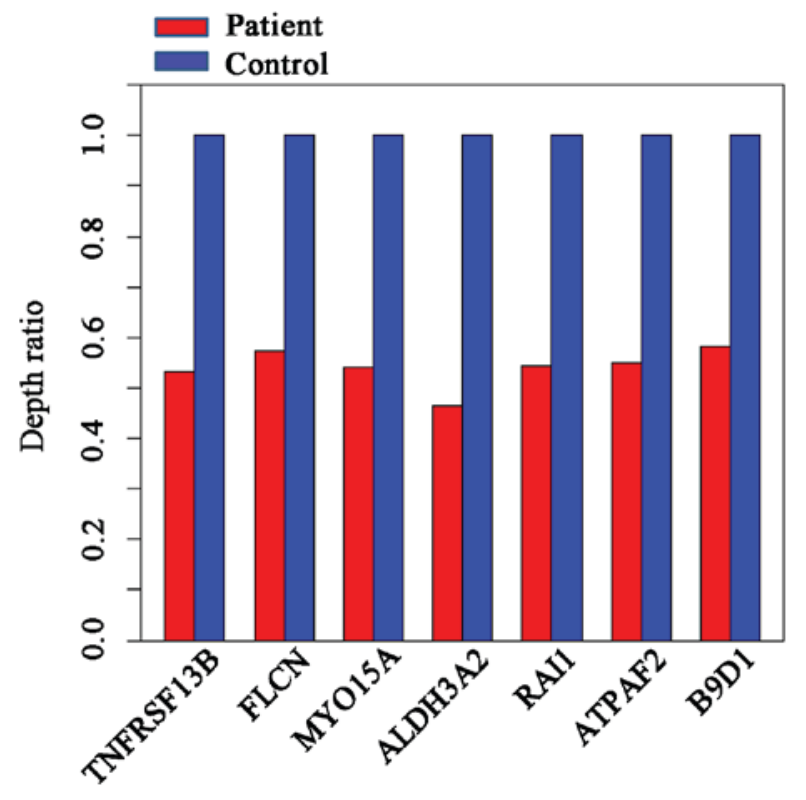

Figure 4. Identification of a heterozygous deletion on $17 \mathrm{p} 11.2$ in the patient with targeted next generation sequencing. The depth ratio (patient:control) of seven genes located on $17 \mathrm{p} 11.2$ was calculated from the designed capture array. The average depth ratio of the patient was approximately 0.55 , indicating a heterozygous deletion. Control, healthy subjects unrelated to the patient.

from $\mathrm{BGI}$ ). If the ratio is 1 , this indicates that no deletion has been detected. A ratio of $\sim 0.5$ indicates the detection of a heterozygous deletion. A ratio of zero indicates a potential homozygous deletion.

The designed capture array identified a 17p11.2 deletion. No single gene mutations were identified. The depth ratio was obtained for seven genes located on 17p11.2; the average depth ratio of the patient was $\sim 0.55$, indicating a heterozygous deletion (Fig. 4). As chromosome 6 was not designed in the capture array, the specific deletion on chromosome 6 was not identified.

$L C S$. To validate the results obtained from the improved NGS method, LCS was additionally performed on the peripheral blood samples of the patient and parents using the Illumina Hiseq2000 (Illumina, Inc.) platform with the population-scale microdeletions and microduplications calling (PSCC) method, as described previously (19). PSCC is a stable and sensitive method for the detection of copy number variation (CNV). PSCC is able to identify deletions with a resolution in the $100 \mathrm{~kb}$ range. It has three modules which include a two-step correction procedure to remove the local GC content bias, a binary segmentation method to locate the candidate microdeletions and microduplications regions and a combined statistics test to estimate the signal reliability. Subsequently, the microdeletions and microduplications are determined.

LCS identified two deletions in the patient, located on 6p21.1 and $17 \mathrm{p} 11.2$ (Fig. 5). The deletion size on 6p21.1 was approximately $172.71 \mathrm{~kb}$ (chromosome 6, 44895251-45077965). The Database of Genomic Variants (dgv.tcag.ca/dgv/app/home) indicated that the identified deletion was not causative. The deletion size on $17 \mathrm{p} 11.2$ was approximately $3.66 \mathrm{Mb}$ (chromosome 17, 16572714-20229256), which is typically

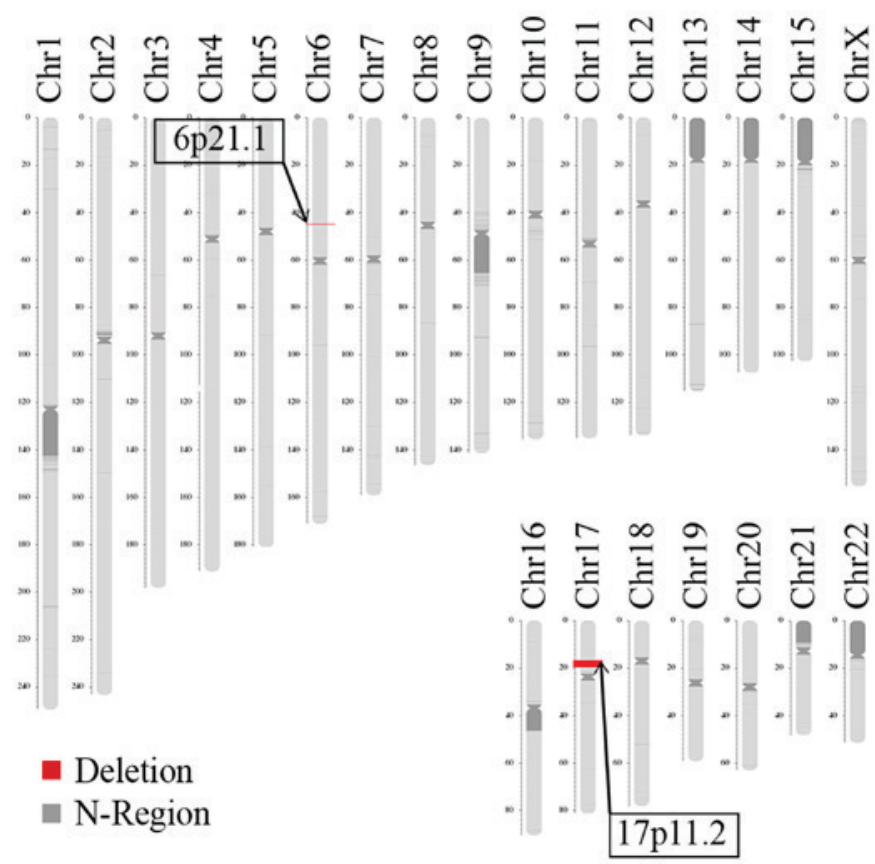

Figure 5. Electrical karyotyping image construction with low coverage whole-genome screening. Red bands indicate the deleted regions. N-region bands indicate the normal regions. Chr, chromosome.

observed in patients with SMS. The LCS results revealed the parents were normal, with no disease-causing microdeletions and microduplications or balanced translocation (data not shown).

\section{Discussion}

Congenital mental retardation is a neurodevelopmental disease with a variety of causes. Genetic factors have significant involvement in the etiology of congenital mental retardation (3). Numerous methods, including CMA and NGS, may be used to detect genetic abnormalities. However, it is difficult to initially select a method to be performed. Using a number of methods increases cost and reduces efficiency. At present, a method to detect genetic abnormalities in one step does not exist. Herein, an improved targeted NGS method was developed to diagnose chromosomal anomalies and single gene mutations in one step at low cost. In the present study, an unknown cause of non-syndromic mental retardation was successfully diagnosed with the improved targeted NGS method. SMS has similar phenotypic features to other syndromes, including 9q34 deletion syndrome, Prader-Willi syndrome, 22q11.2 syndrome, Sotos syndrome and Williams syndrome. Therefore, it may be easily misdiagnosed (14). In the present study, the improved targeted NGS method detected a deletion located in 17p11.2. Thus, the patient was accurately diagnosed with SMS.

The methods of the present study had certain limitations. Chromosomal microdeletions and microduplications were only detected in the targeted captured region, and the whole genome region was not covered. Therefore, breakpoints of chromosome aberration were unable to be detected. Additionally, there was the potential for false negative errors. A future study will improve the scheme to minimize the false 
negative rate, by increasing the targeted captured region and optimizing the information analysis algorithm.

Additionally, fertility guidance was given to the parents of the patient. The origin of the majority of deletions is de novo, and are more rarely attributed to an unbalanced segregation of a parental balanced translocation (20). In the present case, the chromosomes of the asymptomatic parents were normal; no disease-causing microdeletions, microduplications or balanced translocations were detected, indicating that the deletion 17p11.2 was de novo. The incidence of de novo deletions may primarily occur as random chance events during gamete formation or early embryo development. Therefore, the risk of having another affected child may be very low. However, the deletion may be caused by germline mosaicism, a well reported explanation of the possible origin of autosomal dominant and X-linked disorders (21-24). As for de novo deletions, limited information is available in the literature. Rothlisberger et al (20) reported that germline mosaicism is rare, although it may never be excluded as the origin of de novo structural aberrations. Sanchez et al (25) reported that a 15q11.2-q13 deletion in dizygotic twins with Angelman syndrome originated from somatic and germline mosaicism of the mother. The present study did not assess the possibility of germline mosaicism, as it is difficult to obtain gametes. However, prenatal testing should be proposed to this family during genetic counselling to minimize recurrence risks in subsequent gestation.

In summary, targeted NGS is typically used to detect variants of monogenic diseases. In the present study, it was investigated if the currently available targeted NGS was already suitable for the molecular diagnosis of microdeletion. Subsequently, the 17p11.2 deletion was identified by the designed capture array and no single gene mutation was identified. It was demonstrated that this method could successfully identify microdeletion and duplication. Therefore, this improved method has the potential to be developed into a screening panel for effective diagnosis of genetic abnormalities in non-syndromic mental retardation and other congenital anomalies.

\section{Acknowledgements}

Not applicable.

\section{Funding}

This work was supported by Shenzhen Technological Innovation Plan-Technology Development Project (grant no. CXZZ 20130517144604091).

\section{Availability of data and materials}

The datasets generated and/or analyzed during the current study are available from the corresponding author on reasonable request.

\section{Author's contributions}

WW, BM and YY designed the research and produced the first draft of the article. LM and XG performed the experimental studies. XW and DY contributed to drafting and revising the manuscript. HL, YS, XW and DY performed the data analysis.

\section{Ethics approval and consent to participate}

The research was prospectively reviewed and approved by the ethics committee of BGI-Shenzhen (approval no. BGI-IRB 15083; Shenzen, China). Written informed consent for participation in the present study was obtained from the patient's parents.

\section{Consent for publication}

Written informed consent for participation in the present study was obtained from the patient's parents.

\section{Competing interests}

The authors declare that they have no competing interests.

\section{References}

1. Daily DK, Ardinger HH and Holmes GE: Identification and evaluation of mental retardation. Am Fam Physician 61: 1059-1067, 1070,2000

2. Kaur A, Mahajan S and Singh JR: Cytogenetic Profile of Individuals with Mental Retardation. Int J Hum Genet 3: 13-16, 2003.

3. Chelly J, Khelfaoui M, Francis F, Chérif B and Bienvenu T: Genetics and pathophysiology of mental retardation. Eur J Hum Genet 14: 701-713, 2006.

4. Curry CJ, Stevenson RE, Aughton D, Byrne J, Carey JC, Cassidy S, Cunniff C, Graham JM Jr, Jones MC, Kaback MM, et al: Evaluation of mental retardation: Recommendations of a Consensus Conference: American College of Medical Genetics. Am J Med Genet 72: 468-477, 1997.

5. de Vries BB, Pfundt R, Leisink M, Koolen DA, Vissers LE, Janssen IM, Reijmersdal Sv, Nillesen WM, Huys EH, Leeuw Nd, et al: Diagnostic genome profiling in mental retardation. Am J Hum Genet 77: 606-616, 2005.

6. Pinto IP, Minasi LB, da Cruz AS, de Melo AV, da Cruz E Cunha DM, Pereira RR, Ribeiro CL, da Silva CC, de Melo E Silva D and da Cruz AD: A non-syndromic intellectual disability associated with a de novo microdeletion at $7 \mathrm{q}$ and $18 \mathrm{p}$, microduplication at $\mathrm{Xp}$, and $18 \mathrm{q}$ partial trisomy detected using chromosomal microarray analysis approach. Mol Cytogenet 7: 44, 2014.

7. Tucker T, Zahir FR, Griffith M, Delaney A, Chai D, Tsang E, Lemyre E, Dobrzeniecka S, Marra M, Eydoux P, et al: Single exon-resolution targeted chromosomal microarray analysis of known and candidate intellectual disability genes. Eur J Hum Genet 22: 792-800, 2014.

8. Martinez F, Caro-Llopis A, Roselló M, Oltra S, Mayo S, Monfort S and Orellana C: High diagnostic yield of syndromic intellectual disability by targeted next-generation sequencing. J Med Genet 54: 87-92, 2017.

9. Morgan A, Gandin I, Belcaro C, Palumbo P, Palumbo O, Biamino E, Dal Col V, Laurini E, Pricl S, Bosco P, et al: Target sequencing approach intended to discover new mutations in non-syndromic intellectual disability. Mutat Res 781: 32-36, 2015.

10. Ehmke N, Karge S, Buchmann J, Korinth D, Horn D, Reis O and Häßler F: A de novo nonsense mutation in ZBTB18 plus a de novo $15 \mathrm{q} 13.3$ microdeletion in a 6-year-old female. Am J Med Genet A 173: 1251-1256, 2017.

11. Liu Y, Wei X, Kong X, Guo X, Sun Y, Man J, Du L, Zhu H, Qu Z, Tian P, et al: Targeted next-generation sequencing for clinical diagnosis of 561 mendelian diseases. PLoS One 10: e0133636, 2015.

12. Smith AC, McGavran L, Robinson J, Waldstein G, Macfarlane J, Zonona J, Reiss J, Lahr M, Allen L and Magenis E: Interstitial deletion of (17)(p11.2p11.2) in nine patients. Am J Med Genet 24: 393-414, 1986. 
13. Girirajan S, Elsas LJ II, Devriendt K and Elsea SH: RAI1 variations in Smith-Magenis syndrome patients without $17 \mathrm{p} 11.2$ deletions. J Med Genet 42: 820-828, 2005.

14. Elsea SH and Girirajan S: Smith-Magenis syndrome. Eur J Hum Genet 16: 412-421, 2008.

15. Slager RE, Newton TL, Vlangos CN, Finucane B and Elsea SH: Mutations in RAI1 associated with Smith-Magenis syndrome. Nat Genet 33: 466-468, 2003.

16. Seabright M: A rapid banding technique for human chromosomes. Lancet 2: 971-972, 1971.

17. Magi A, Tattini L, Pippucci T, Torricelli $\mathrm{F}$ and Benelli $\mathrm{M}$ Read count approach for DNA copy number variants detection. Bioinformatics 28: 470-478, 2012

18. Bragin E, Chatzimichali EA, Wright CF, Hurles ME, Firth HV, Bevan AP and Swaminathan GJ: DECIPHER: Database for the interpretation of phenotype-linked plausibly pathogenic sequence and copy-number variation. Nucleic Acids Res 42 (Database issue): D993-D1000, 2014.

19. Li X, Chen S, Xie W, Vogel I, Choy KW, Chen F, Christensen R, Zhang C, Ge H, Jiang H, et al: PSCC: Sensitive and reliable population-scale copy number variation detection method based on low coverage sequencing. PLoS One 9: e85096, 2014.
20. Röthlisberger B and Kotzot D: Recurrence risk in de novo structural chromosomal rearrangements. Am J Med Genet A 143A: 1708-1714, 2007.

21. Wilton SD, Chandler DC, Kakulas BA and Laing NG: Identification of a point mutation and germinal mosaicism in a Duchenne muscular dystrophy family. Hum Mutat 3: 133-140, 1994.

22. Slavin TP, Lazebnik N, Clark DM, Vengoechea J, Cohen L, Kaur M,Konczal L, Crowe CA, Corteville JE, Nowaczyk MJ, et al: Germline mosaicism in Cornelia de Lange syndrome. Am J Med Genet A 158A: 1481-1485, 2012.

23. Bermúdez-López C, García-de Teresa B, González-del Angel A and Alcántara-Ortigoza MA. Germinal mosaicism in a sample of families with Duchenne/Becker muscular dystrophy with partial deletions in the DMD gene. Genet Test Mol Biomarkers 18: 93-97, 2014

24. Miyagawa M, Nishio SY, Hattori M, Takumi Y and Usami S: Germinal mosaicism in a family with BO syndrome. Ann Otol Rhinol Laryngol 124 (Suppl 1): 118S-122S, 2015.

25. Sánchez J, Fernández R, Madruga M, Bernabeu-Wittel J, Antiñolo G and Borrego S: Somatic and germ-line mosaicism of deletion 15q11.2-q13 in a mother of dyzigotic twins with Angelman syndrome. Am J Med Genet A 164A: 370-376, 2014. 